Supporting information for the article:

\title{
Blood-Brain Barrier Penetrating Luminescent Conjugates Based on Cyclometalated Platinum(II) Complexes
}

Anastasia I. Solomatina, ${ }^{\dagger}$ Aleksandra D. Slobodina, ${ }^{\ddagger}$ Elena V. Ryabova, ${ }^{\ddagger}$ Olga I. Bolshakova, ${ }^{\ddagger}$ Pavel S. Chelushkin, ${ }^{\dagger}$ Svetlana V. Sarantseva, ${ }^{*}$, Sergey P. Tunik ${ }^{*}, \dagger$

†Institute of Chemistry, St. Petersburg State University, St. Petersburg, 198504, Russia

‡ Petersburg Nuclear Physics Institute named by B.P. Konstantinov of National Research Centre «Kurchatov Institute», Gatchina, 188300, Russia

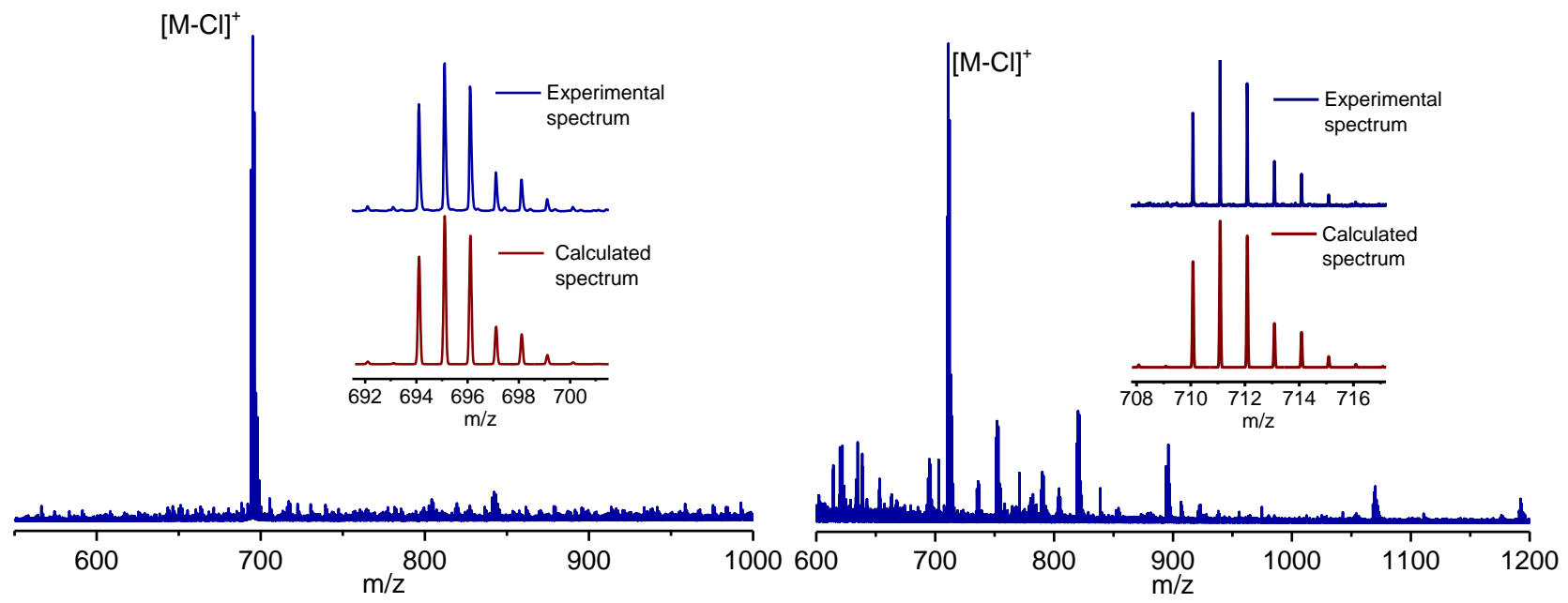

Figure S1. ESI+ mass-spectra of complexes Pt2 and Pt3.

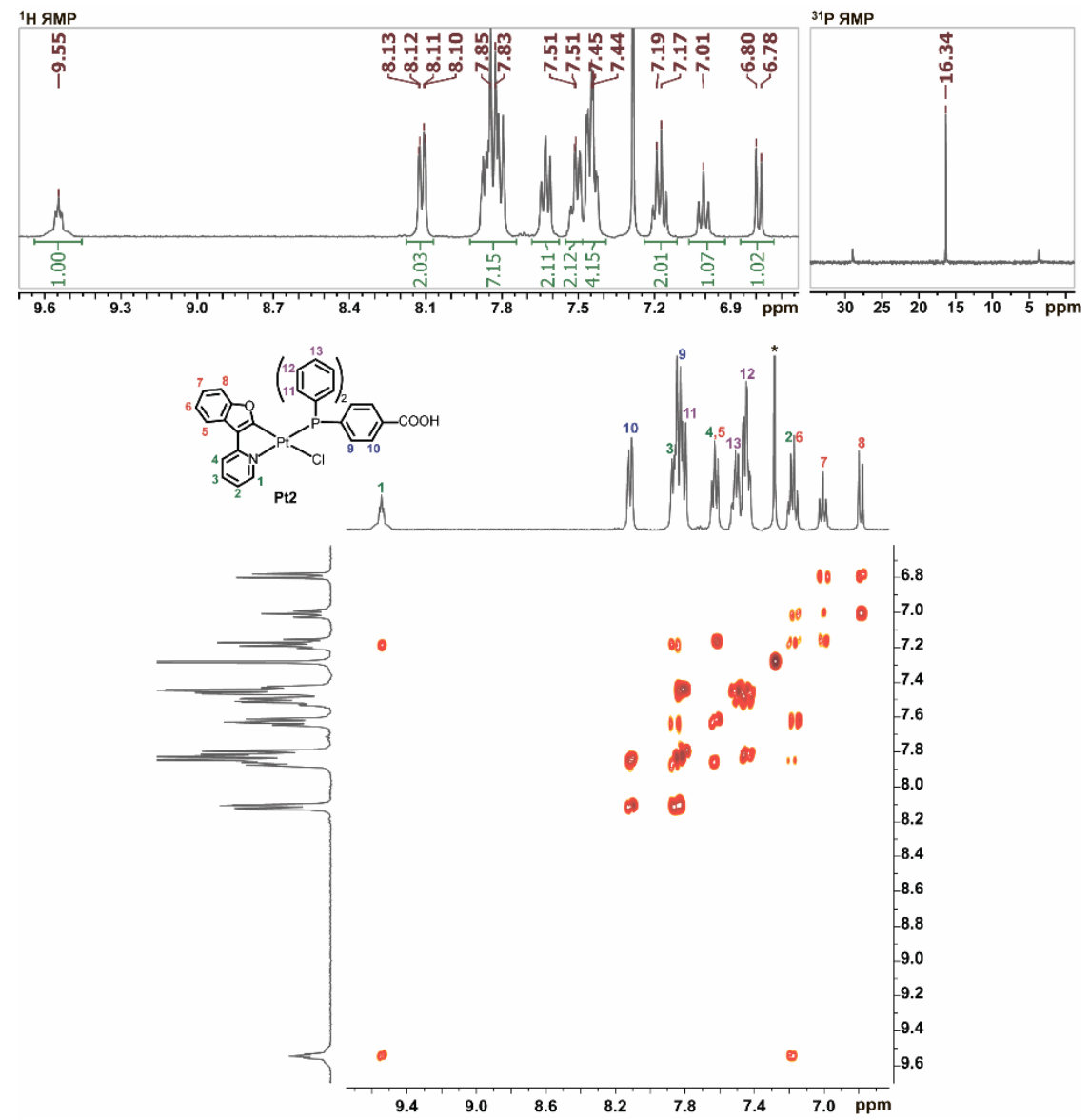

Figure S2. ${ }^{1} \mathrm{H},{ }^{1} \mathrm{H}-{ }^{1} \mathrm{H}$ COSY, and ${ }^{31} \mathrm{P}$ NMR spectra of complex Pt2 in $\mathrm{CDCl}_{3}, 298 \mathrm{~K}$. 

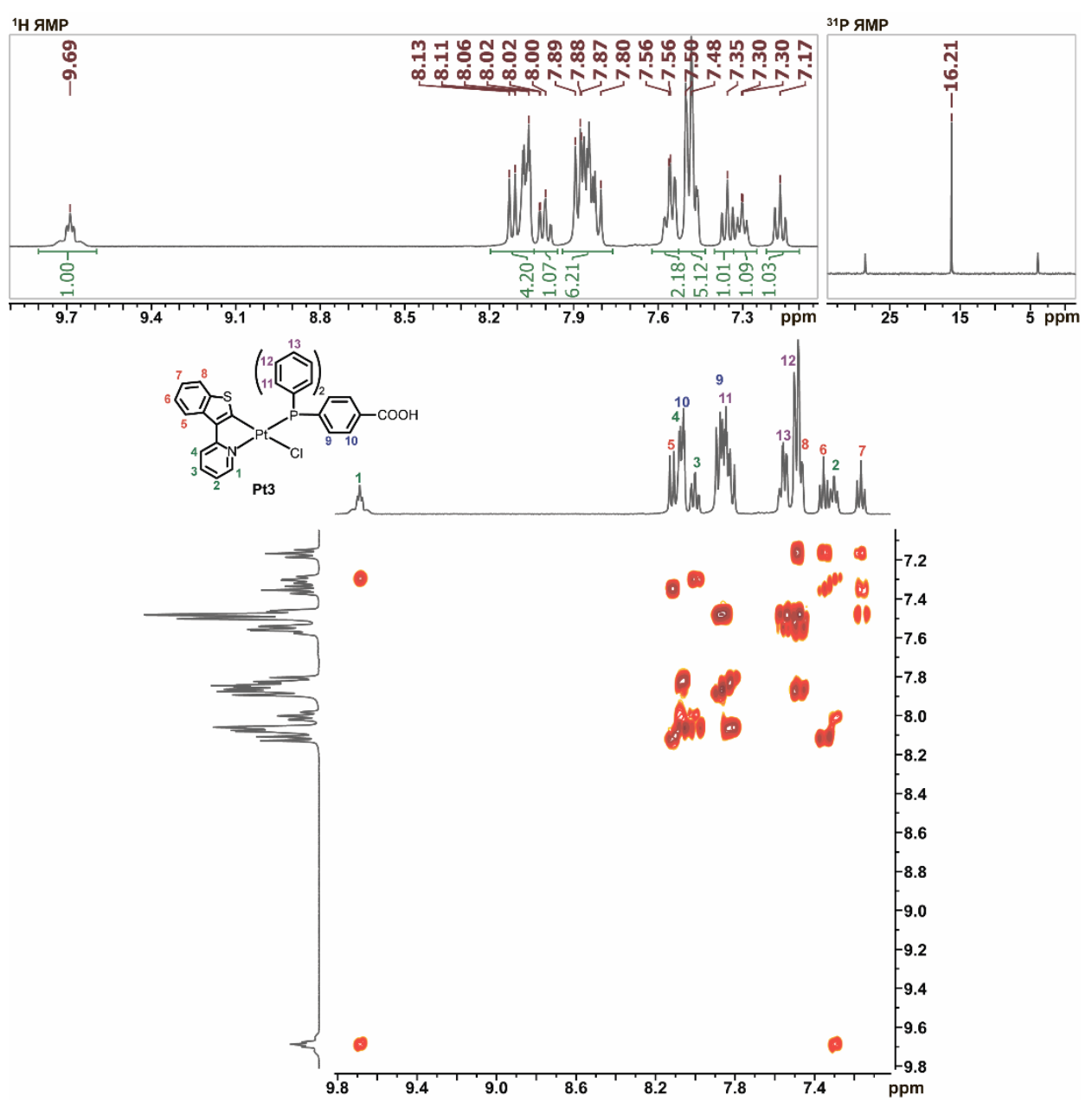

Figure S3. ${ }^{1} \mathrm{H},{ }^{1} \mathrm{H}-{ }^{1} \mathrm{H}$ COSY, and ${ }^{31} \mathrm{P} N M R$ spectra of complex Pt3 in $\mathrm{CD}_{2} \mathrm{Cl}_{2}, 298 \mathrm{~K}$.

A

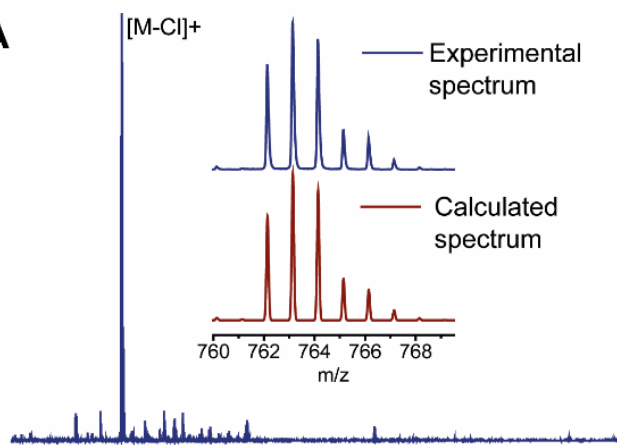

B

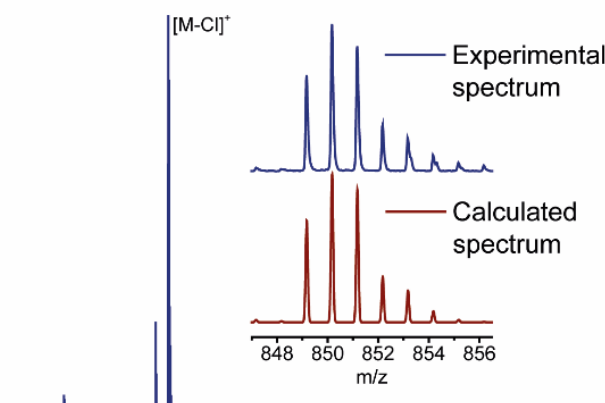
$\begin{array}{llllllllllllllllllll}600 & 700 & 800 & 900 & 1000 & 1100 & 1200 & 1300 & 1400 & 1500 & 600 & 700 & 800 & 900 & 1000 & 1100 & 1200 & 1300 & 1400 & 1500\end{array}$

C

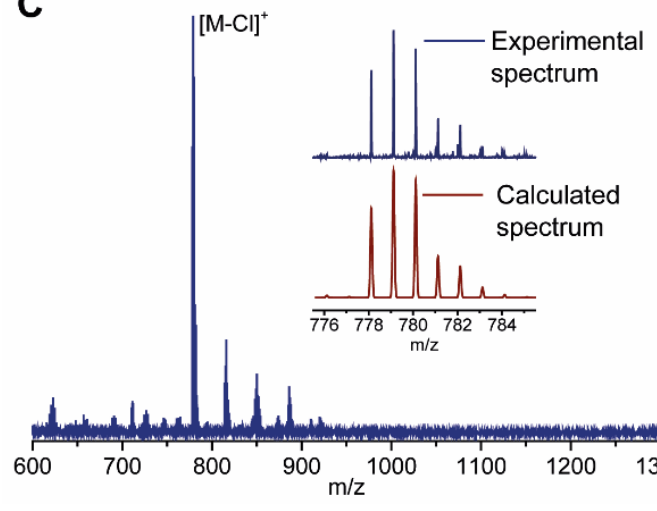

D $\quad \mid[\mathrm{M}-\mathrm{Cl}]^{+}$

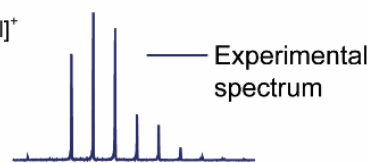

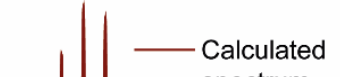
spectrum

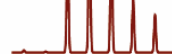

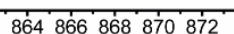
$\mathrm{m} / \mathrm{z}$

Figure S4. ESI+ mass-spectra of complexes Pt2-Im (A) and Pt2-His (B), Pt3-Im (C), and Pt3-His (D). 

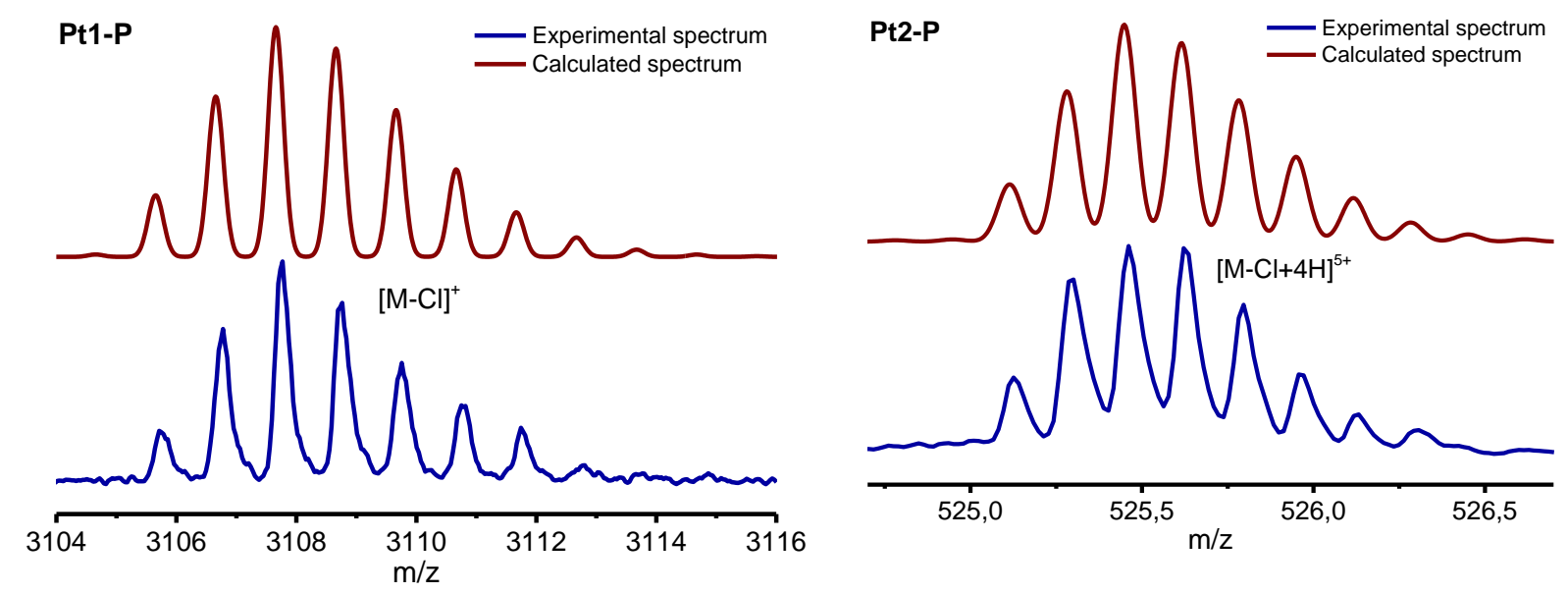

Figure S5. ESI+ mass-spectra of conjugates Pt1-P and Pt2-P.
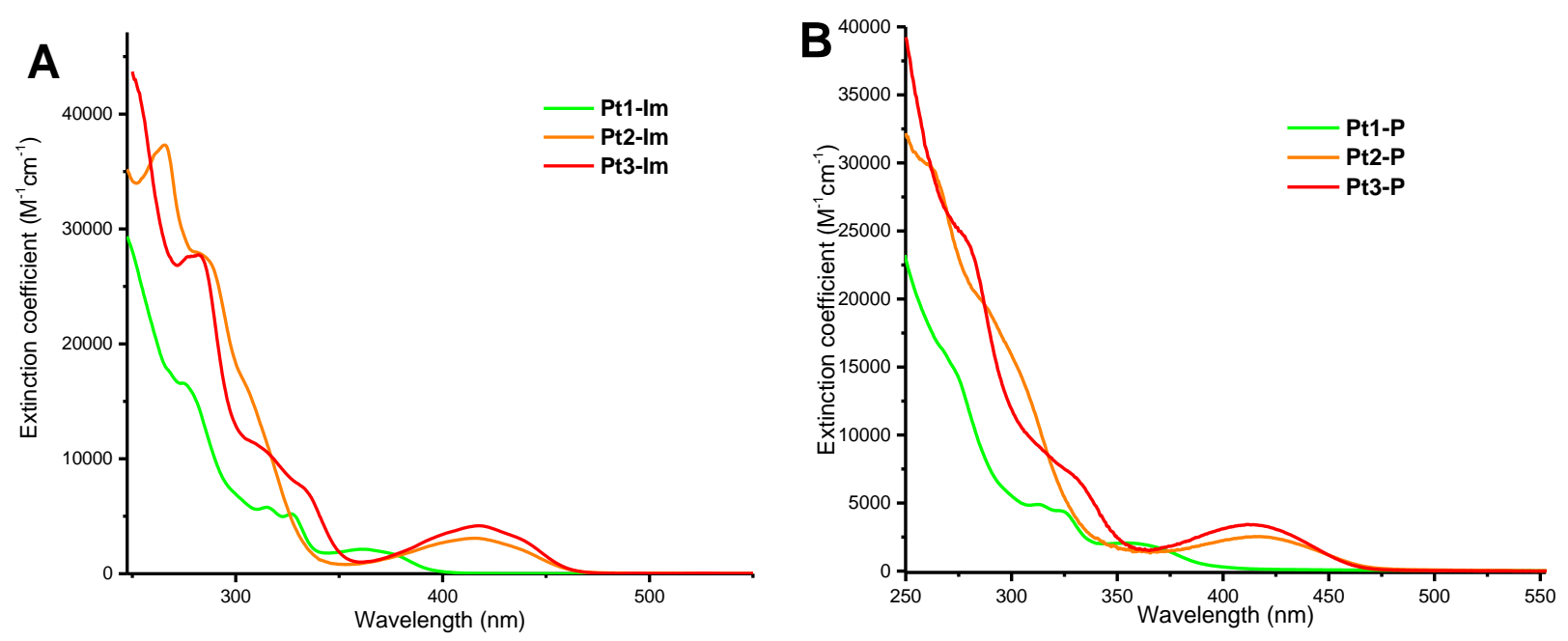

Figure S6. Absorption spectra of (A) complexes Pt1-Im - Pt3-Im in DCM and (B) conjugates Pt1-P - Pt3-P in water.

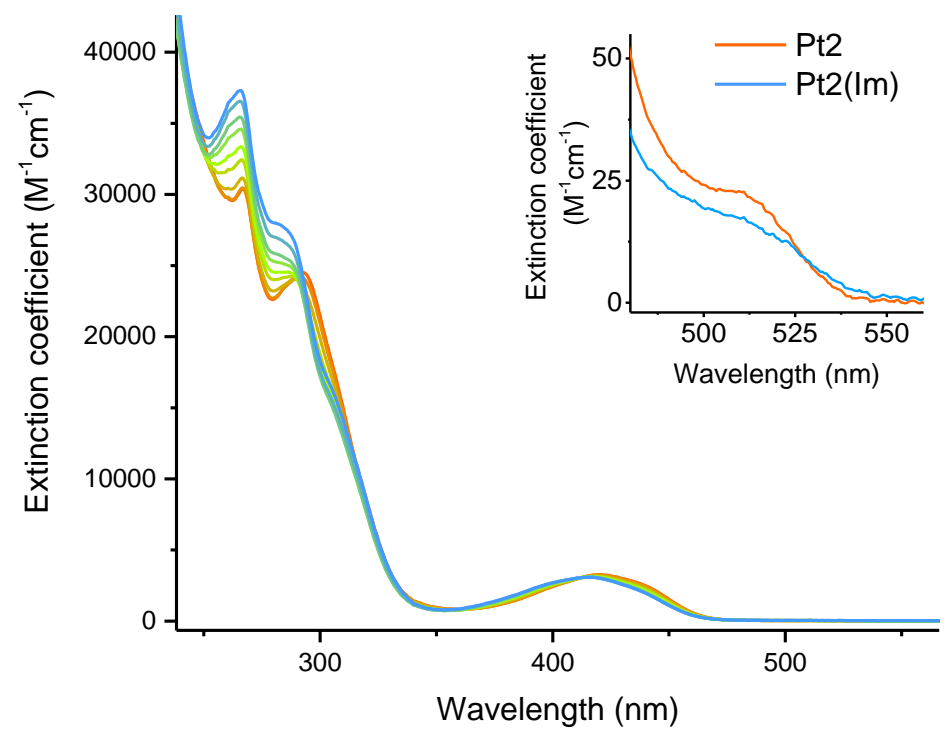

Figure S7. Titration of Pt2 solution with imidazole, DCM, RT. 

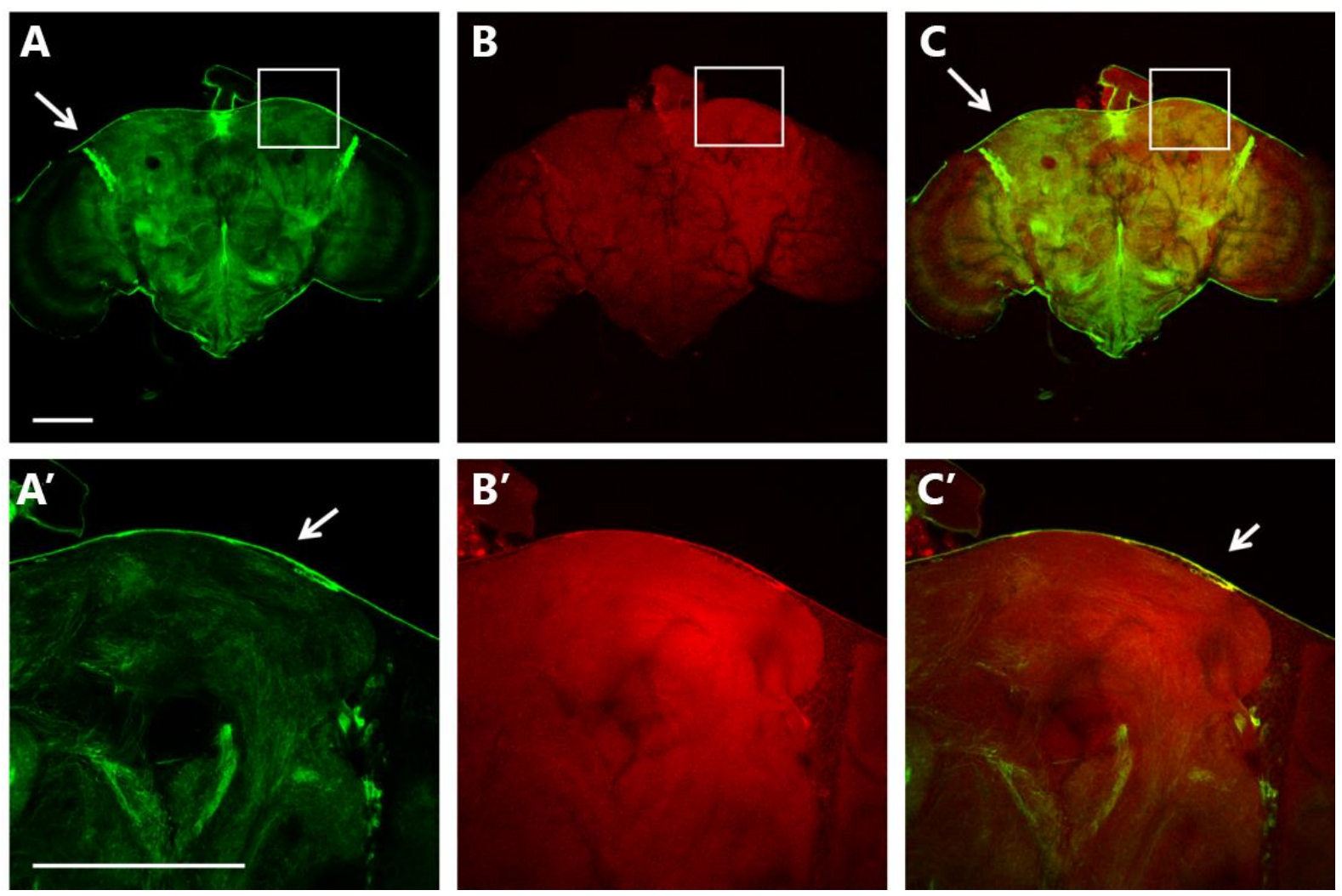

Figure S8. Internalization of Pt3-P in the brain of Drosophila melanogaster Canton $S$ in 2 h after injection, $A$ and $A^{\prime}-B B B$ visualization (denoted by arrows), $B$ and $B^{\prime}-P t 3-P$, and $C$ and $C^{\prime}-$ overlay of the images. Scale bar $-100 \mu m$.
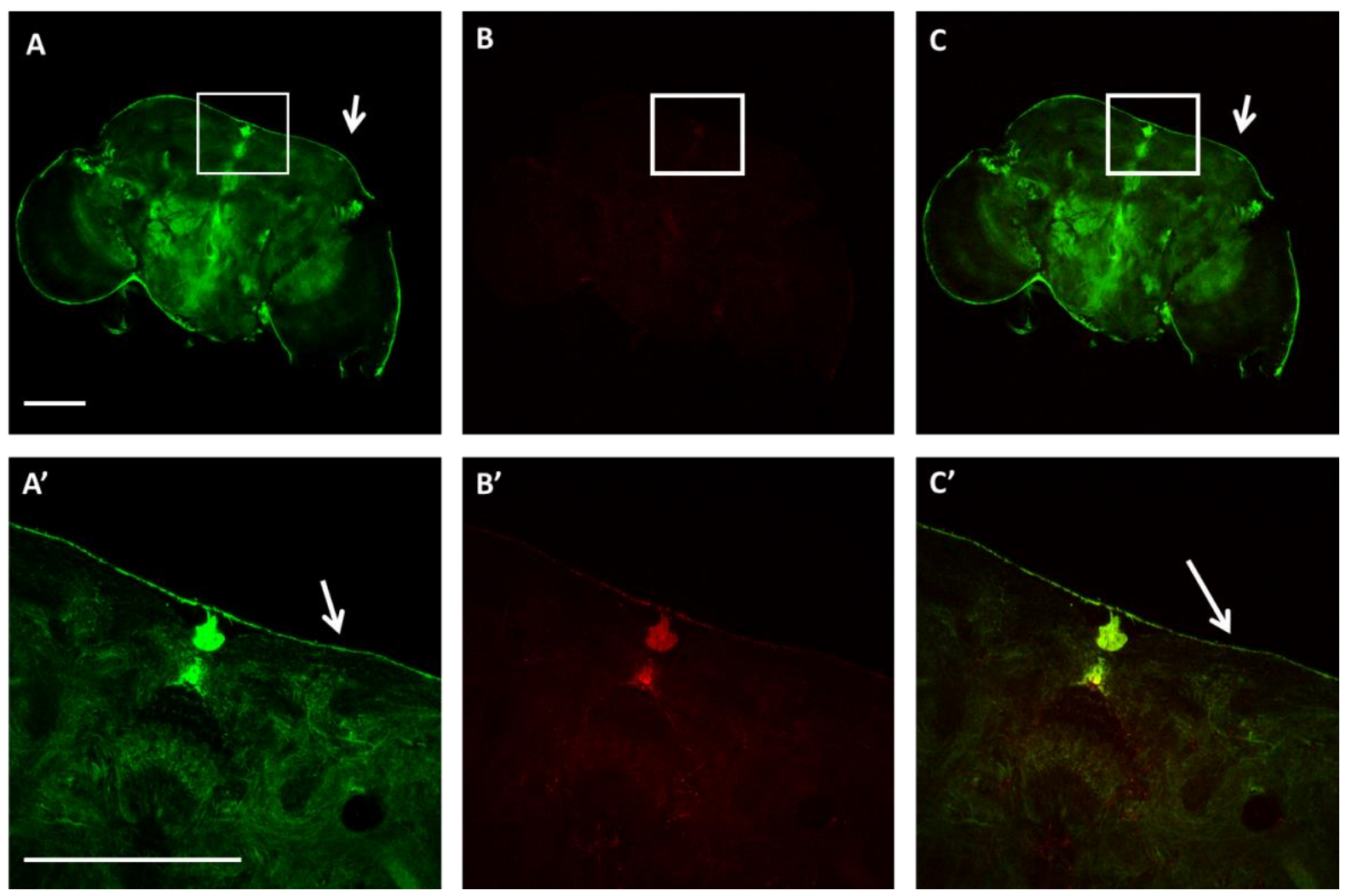

Figure S9. Control. Visualization of the brain of Drosophila melanogaster Canton $S$ in $2 \mathrm{~h}$ after injection of Ringer solution, $A$ and $A^{\prime}-$ $B B B$ visualization (denoted by arrows), $B$ and $B^{\prime}-$ emission in red channel, and C and $C^{\prime}$ - overlay of the images. Scale bar -100 $\mu m$. 

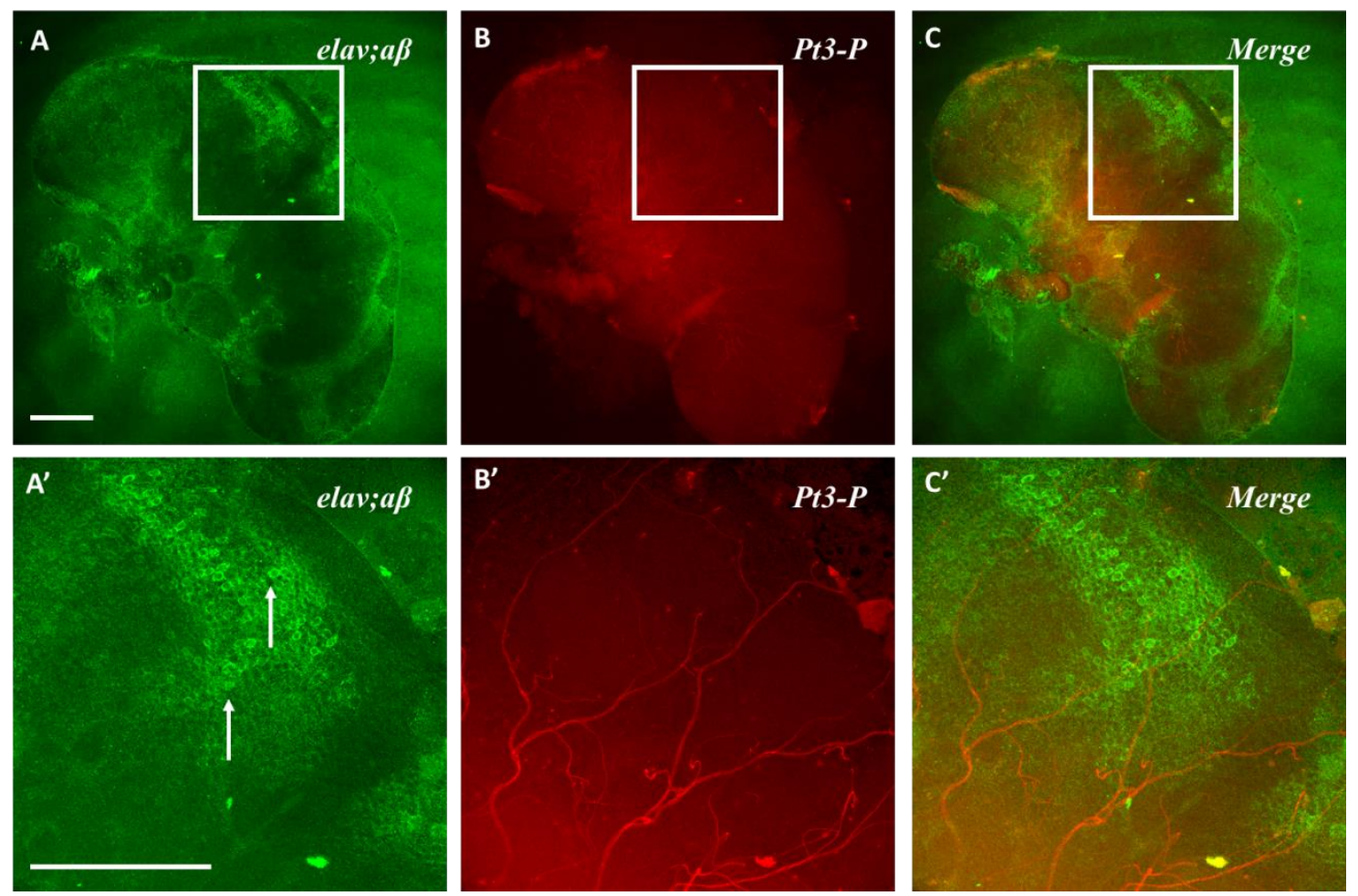

Figure S10. Localization of $\beta$-amyloid peptide ( $A$ and $\left.A^{\prime}\right)$, conjugate Pt3-P ( $B$ and $\left.B^{\prime}\right)$ in the brain of Drosophila melanogaster $A B$. Arrows denote amyloid deposits. C and $C^{\prime}$ - overlay of two channels. Scale bar- $100 \mu \mathrm{M}$.
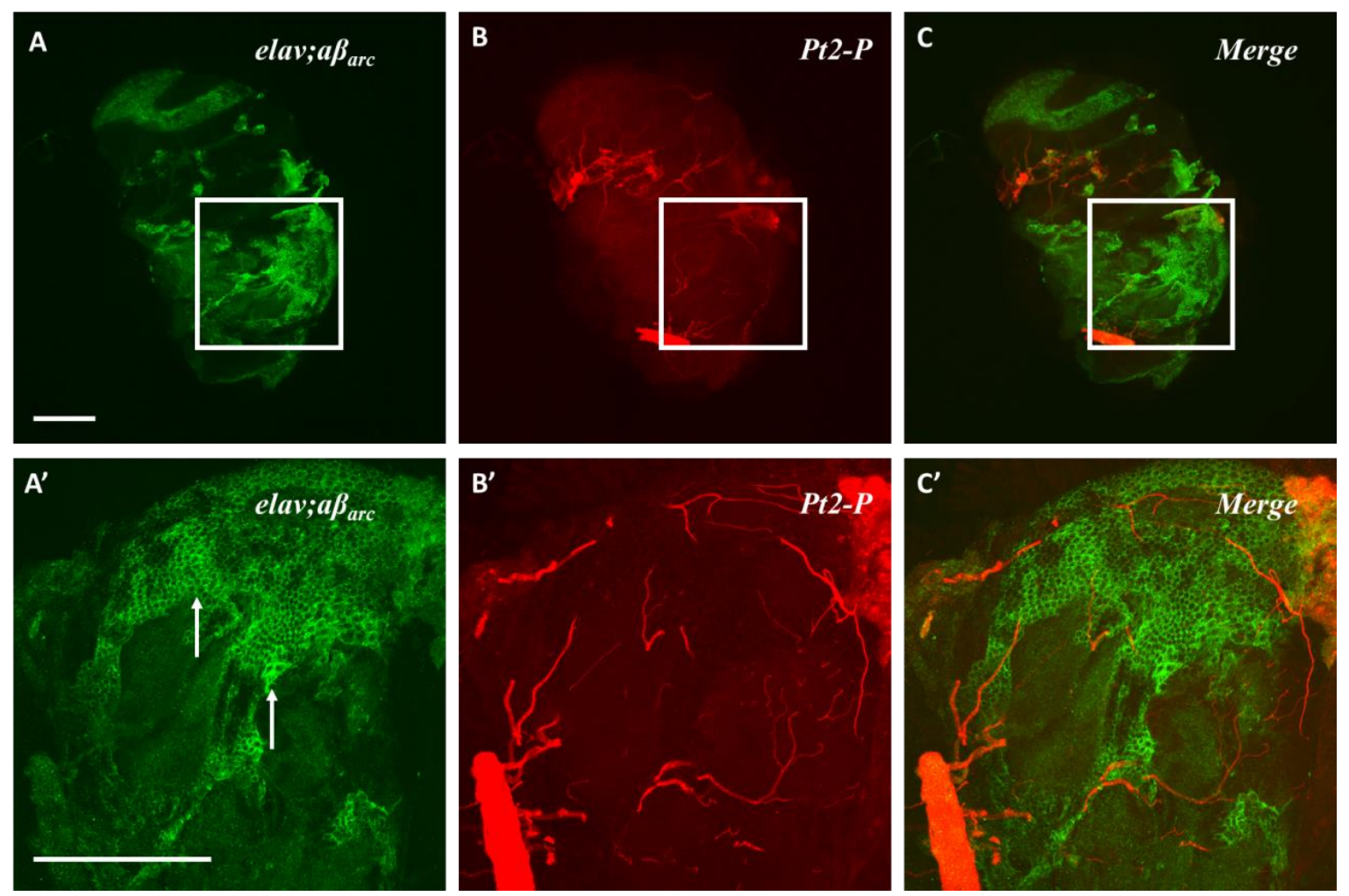

Figure S11. Localization of $\beta$-amyloid peptide ( $A$ and $\left.A^{\prime}\right)$, conjugate Pt2-P ( $B$ and $\left.B^{\prime}\right)$ in the brain of Drosophila melanogaster $A B$ Arctic. Arrows denote amyloid deposits. $C$ and $C^{\prime}-$ overlay of two channels. Scale bar $-100 \mu \mathrm{M}$. 

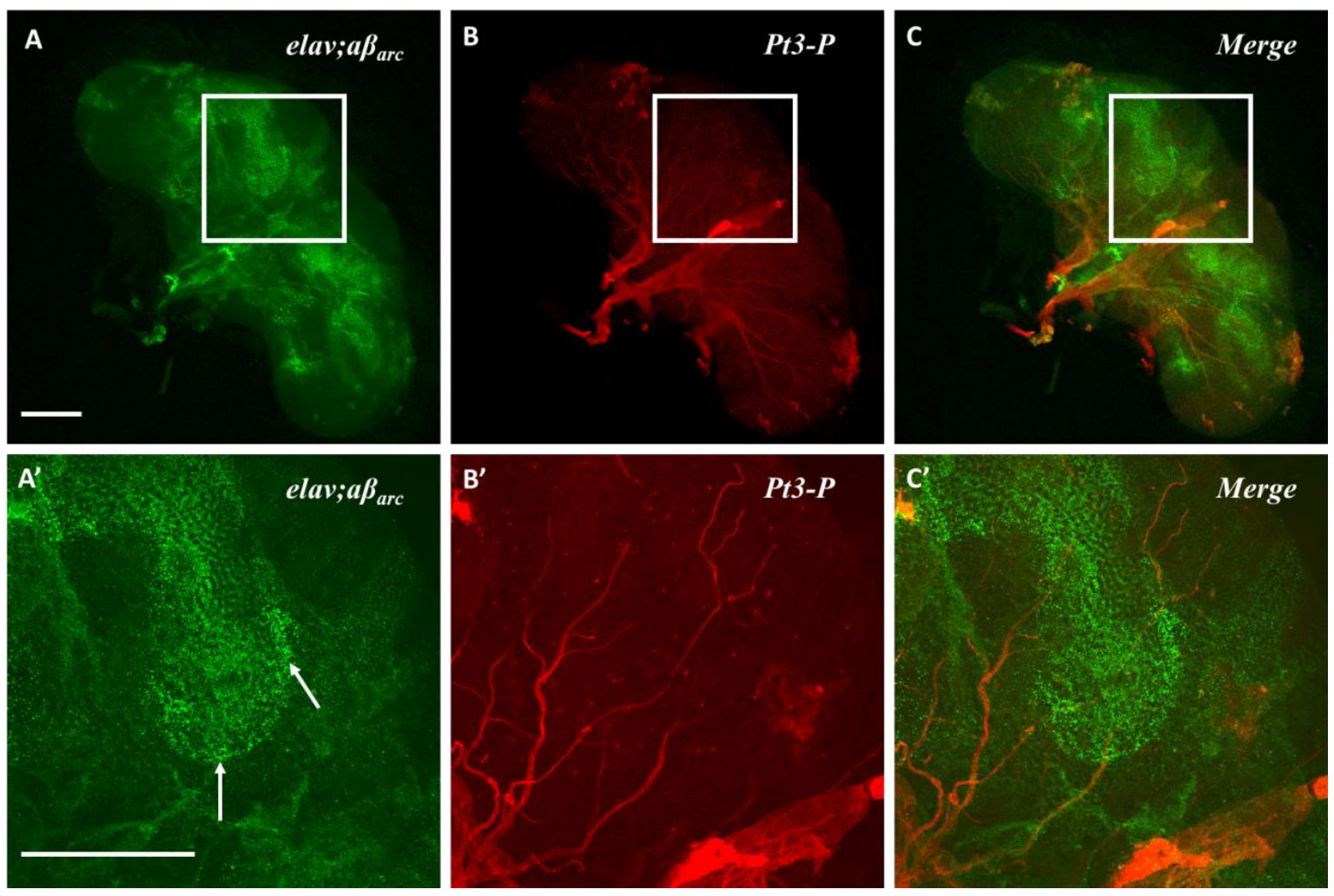

Figure S12. Localization of $\beta$-amyloid peptide ( $A$ and $\left.A^{\prime}\right)$, conjugate Pt3-P ( $B$ and $\left.B^{\prime}\right)$ in the brain of Drosophila melanogaster $A B$ Arctic. Arrows denote amyloid deposits. $C$ and $C^{\prime}$ - overlay of two channels. Scale bar $-100 \mu \mathrm{M}$.
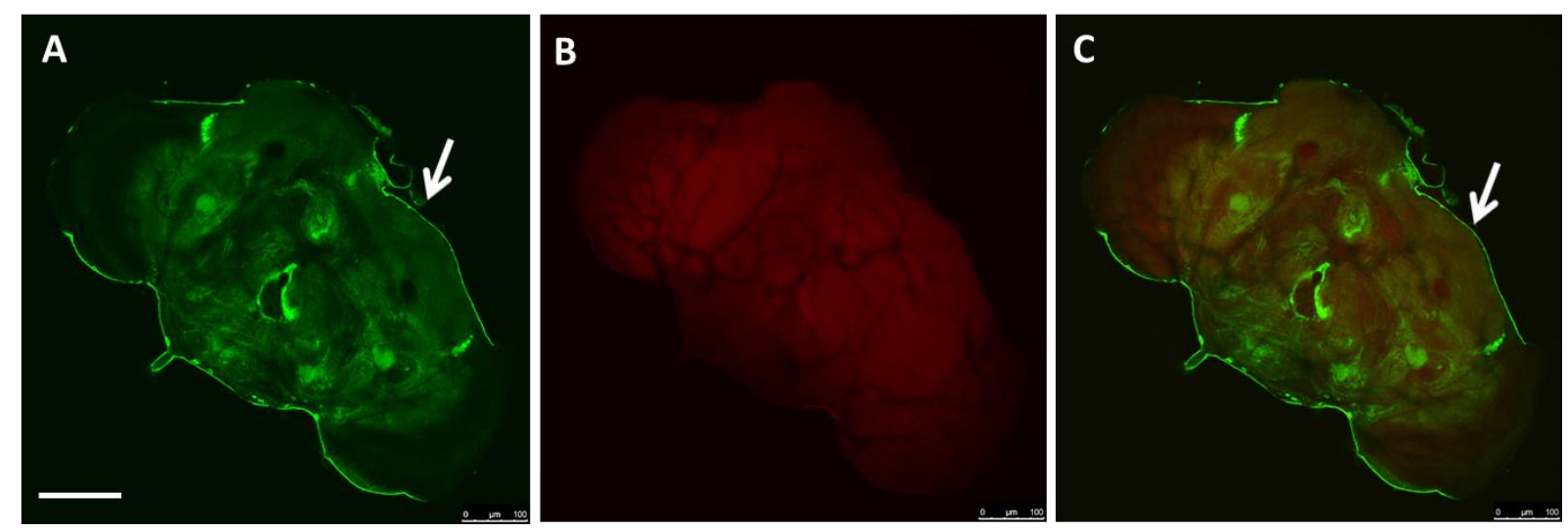

Figure S13. Pt2 does not pass through the BBB of Drosophila melanogaster Canton $S$ in 2 h after injection, $A$ - BBB visualization (denoted by arrows), B - Pt2, and C - overlay of the images. Scale bar $-100 \mu \mathrm{m}$.
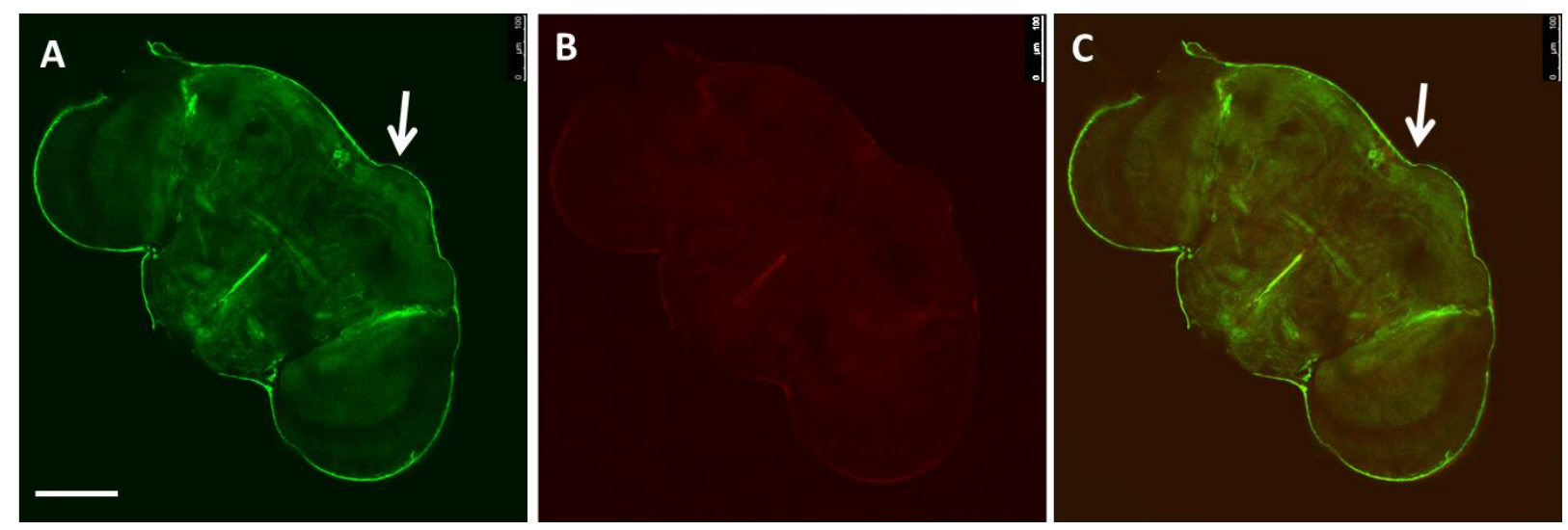

Figure S14. Pt3 does not pass through the BBB of Drosophila melanogaster Canton $S$ in 2 h after injection, A - BBB visualization (denoted by arrows), B - Pt3, and C - overlay of the images. Scale bar $-100 \mu \mathrm{m}$. 


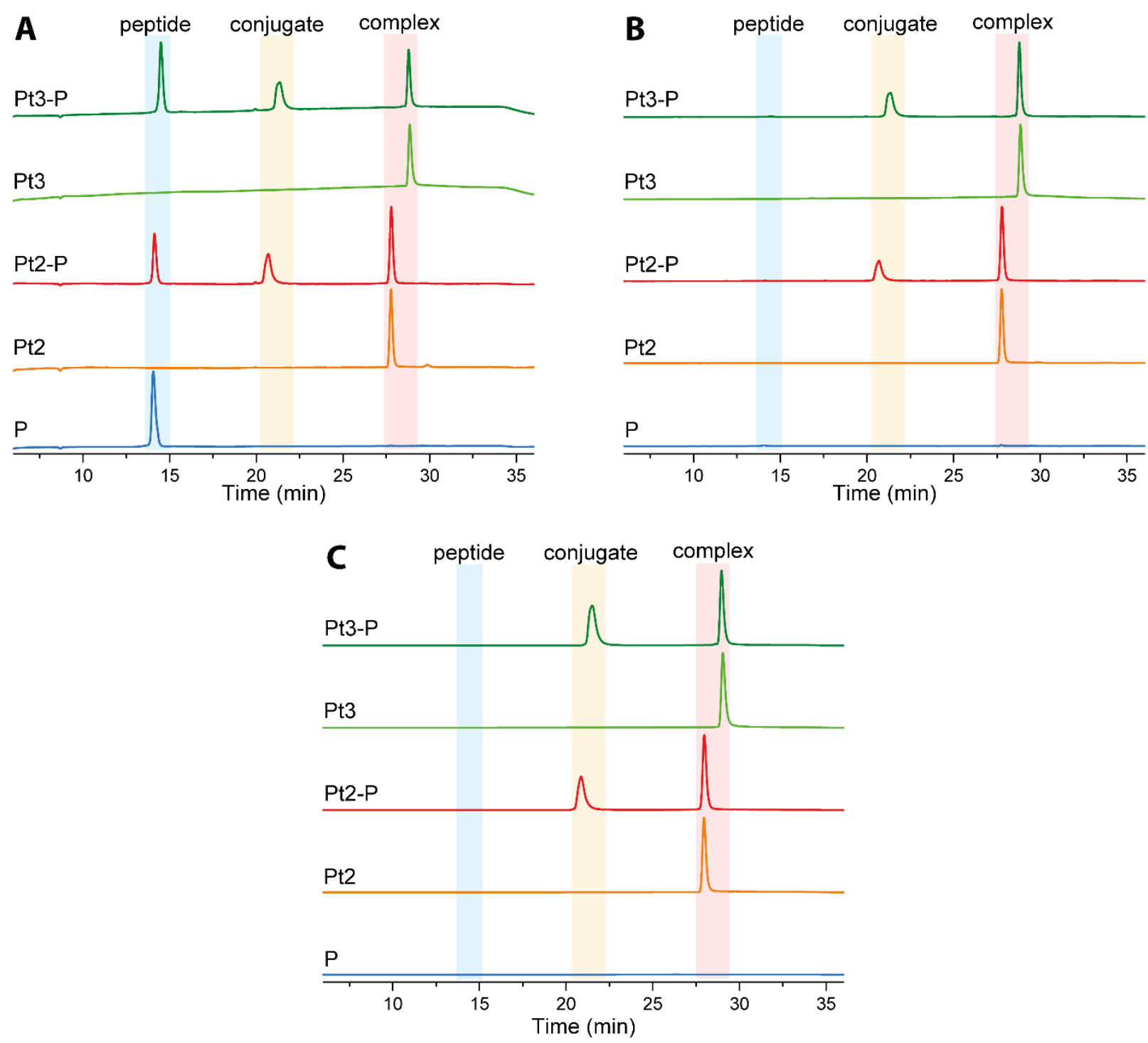

Figure S15. HPLC chromatogram for Pt2-P and Pt3-P, gradient mode 5-90\% $\mathrm{CH}_{3} \mathrm{OH}$ in water, $0.1 \% \mathrm{TFA}, 0.3 \mathrm{~mL} / \mathrm{min}$. Column: Zorbax Eclipse XDB-C18 150*2.1 mm, $5 \mu \mathrm{m}$. Sample concentration $0.3 \mu \mathrm{M}$, volume $5 \mu \mathrm{L}$. Detection of absorption at $214 \mathrm{~nm}$ (A) and $280 \mathrm{~nm}$ (B), and (C) detection of emission at $570 \mathrm{~nm}\left(\lambda_{e x}=410 \mathrm{~nm}\right)$.
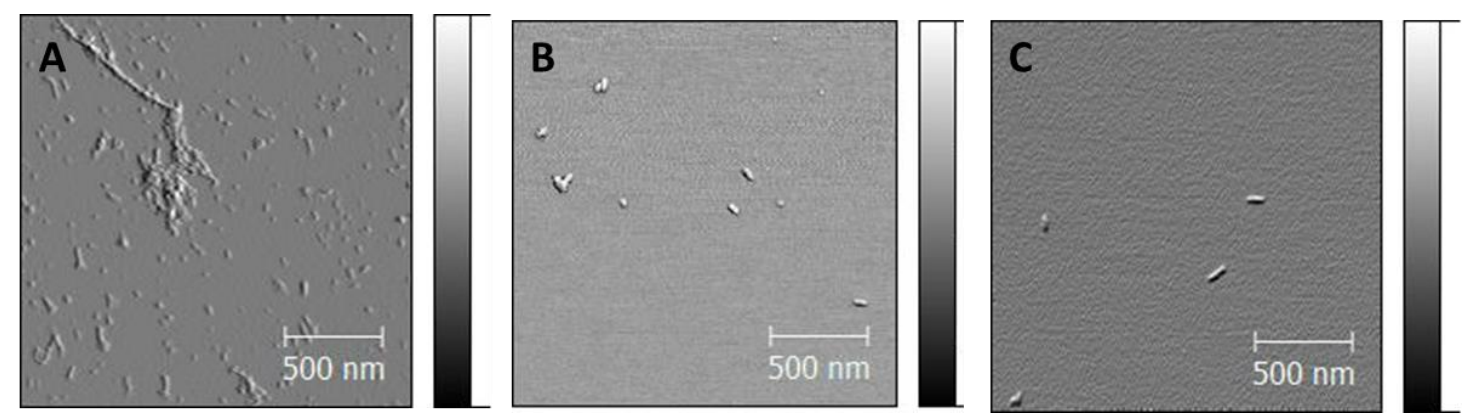

Figure S16. AFM images of aggregates formed during incubation of $A \beta 42(A)$, after joint incubation of $A \beta 42$ and Pt2-P (B), Aß42 and Pt3-P (C). 